He then passes on to a consideration of the diagnosis and management of these mild cases of diphtheria. The diagnosis of catarrhal diphtheria depends entirely upon bacteriological examinations. Care should therefore be taken to have such an examination carried out in every case where there is reason to suspect the possibility of diphtheritic infection, and means should be provided by the public to facilitate this custom. With regard to the management, the immediate concern is to educate the laity to a proper appreciation of the serious importance and danger to others of catarrhal diphtheria. The next step would be to include the condition in the list of notifiable diseases under its own name, and to extend to it the same sanitary regulations which relate to the management of the well marked types of diphtheria.

$$
1 \text { Medical News, Nov. } 21 .
$$

\section{SYPHILITIC JOINT DISEASE.}

Mr. Percy Patos, ${ }^{1}$ writing on this subject, states that though uncommon, the condition occurs somewhat more frequently than is generally supposed and is often overlooked or mistaken for some other affection. Of joint lesions in secondary syphilis he describes three varieties (1) arthralgia, (2) synovitis, and (3) hydrarthrosis. The first, in which pain increasing at night and without limitation of movement is almost the only symptom, occurs early, sometimes before the secondary eruption. Synovitis, a more common variety, may simulate rheumatism or come on without warning as an effusion with some local tenderness and pain which is not increased by movement. Hydrarthrosis in secondary syphilis nearly always affects the knee and occurs late in this stage ; its onset is gradual and painless. Five varieties of tertiary syphilitic joint lesion are described. (1) Synovitis, either acute or chronic, in which the symptoms are those of a painless osteoarthritis. (2) Gummatous synovitis, in which condition there may be either much or little fluid ; the synovial membrane is puckered and lumpy, pain is chiefly at night and not starting in character; relapses are frequent. (3) In some cases bones or cartilages are primarily diseased. Though there is little pain there is much limitation of movement, and thickening of bone ends with lipping may be a marked feature. Ankylosis is to be feared if this condition is not recognised and treated early. (4) A gumma near a joint may perforate the joint and cause secondary effusion. A much more serious complication sometimes occurs when a gumma bursts externally and also perforates the joint causing septic arthritis followed by destruction of the joint. (5) Ankylosis may occur in any case of gummatous joint affection which is not recognised and treated early, and especially when the joint is kept rigidly at rest.

In hereditary syphilis the following six varieties of joint disease have been described:-(1) Simple synovial effusion, which is fairly common, affects the knee, and is often associated with interstitial keratitis. (2) Joint complication of syphilitic epiphysitis is characterised by much boggy swelling round the joint and loss of function in the limb. (3) Primary gummatous affection of the synovial membrane; and (5) osteitis and gummatous synovitis which have the same symptoms in the hereditary as in the acquired disease. (4) Osteitis, in which the ends of the bones are enlarged and fluid is poured out into the synovial cavity, is a painless condition, amenable to treatment, frequently relapsing. (6) Deforming arthritis is rarely seen. Osteophytic outgrowths limit the joint movement and sometimes similate dislocation rather than disease.

The special diagnostic points in all syphilitic arthropathies, apart from other evidence of syphilis, are the slight pain on movement, the mild constitutional disturbance, the fact that the knee is the joint most often affected, and the satisfactory results of anti-syphilitic treatment.

Treatment consists in the exhibition of mercury and iodides, counter-irritation, passive and active movement, and massage.

1 British Medical Journal, Nov. 28, 1903.

\title{
Diseases of the Heart and Circulation.
}

Stokes-Adams Disease.-Many interesting cases of this rare disease are recorded by Osler, ${ }^{1}$ together with a discussion of its nature and treatment. The disease is a clinical condition characterised by (1) a disturbance in the automatic mechanism of the heart - true bradycardia, hemisystole (false bradycardia) and allorythmia; (2) nervous symptoms-vertigo, syncope, pseudo-apoplexy and epileptiform attacks; and (3) secondary symptoms - Cheyne-Stokes breathing, cardiac asthma, angina pectoris and the vaso-motor accompaniment of profound heart shock. The post-mortem changes are not constant, in a few cases coarse lesions of the nervous system have been found, in a few no lesions whatever, but generally arterio sclerosis is present. A slow pulse is found in some few individuals as a physiological condition; in organic disease of the nervous system, in toxic conditions, e.g. diphtheria and typhoid fever, digitalis and tobacco poisoning, and in uræmia and jaundice, and in cardiac and cardio-vascular lesions. Stokes-Adams disease is a symptom-complex, not one with constant anatomical lesions or a uniform etiology. The cases may be divided into three categories. (1) Post-febrile groupfollowing some acute affection, such as typhoid fever, pneumonia or rheumatism, bradycardia may occur with vertigo, syncope, or epileptiform seizures. This form is the most hopeful, though the first attack may prove fatal. (2) Neuritic group-generally with coarse lesions of the nervous system, most often extrinsic. (3) Arterio-sclerotic group-comprising by far the majority of cases which have been recorded. The diagnosis in the severe form is usually simple, and is only difficult in cases of aggravated neurasthenia associated with bradycardia and vertigo. The cardio-vascular features are of great interest. The bradycardia is usually a true one, i.e., the heart beats and pulse beats are of equal rate. In a few cases this true bradycardia alternated with a false one, $i$ e., one in which the pulse was slow because only every alternate beat reached the wrist. The pulse is usually full, strong and regular; the infrequency seems to be due to prolongation of the diastole, the systole being sharp and quick. The rate may be 